\title{
Medication adherence during adjunct therapy with statins and ACE inhibitors in adolescents with type 1 diabetes
}

\author{
Elżbieta Niechciał, PhD ${ }^{1}$, Carlo L. Acerini, MD $^{2}$, Scott T. Chiesa, $\mathrm{PhD}^{3}$, Tracey Stevens, \\ BSc $^{2}$, R. Neil Dalton, PhD $^{4}$, Denis Daneman, MD ${ }^{5}$, John E. Deanfield, MD ${ }^{3}$, Timothy W. \\ Jones, MD ${ }^{6}$, Farid H. Mahmud, MD ${ }^{5}$, Sally M. Marshall, MD ${ }^{7}$, H. Andrew W. Neil, DSc ${ }^{8}$, David \\ B Dunger, MD $^{2,9}$, M. Loredana Marcovecchio, MD, $\mathrm{PhD}^{2}$ on behalf of the Adolescent type 1 \\ Diabetes cardio-renal Intervention Trial (AdDIT) study group
}

\begin{abstract}
${ }^{1}$ Department of Pediatric Diabetes, Endocrinology and Obesity, Poznan University of Medical Sciences, Poznan, Poland ${ }^{2}$ Department of Paediatrics, University of Cambridge, Cambridge, UK ${ }^{3}$ Vascular Physiology Unit, UCL Institute of Cardiovascular Science, London, UK ${ }^{4}$ Evelina Children's Hospital, Guy's and St Thomas' NHS Foundation Trust, London, UK ${ }^{5}$ Department of Paediatrics, The Hospital for Sick Children and University of Toronto, Toronto, Ontario, Canada ${ }^{6}$ Telethon Kids Institute, The University of Western Australia, Perth, Australia ${ }^{7}$ Institute of Cellular Medicine (Diabetes), Faculty of Clinical Medical Sciences, Newcastle University, Newcastle upon Tyne, UK ${ }^{8}$ Oxford Centre for Diabetes, Endocrinology \& Metabolism, University of Oxford, Oxford, UK ${ }^{9}$ Wellcome Trust-MRC Institute of Metabolic Science, University of Cambridge, Cambridge, UK
\end{abstract}

\begin{abstract}
Objective-Suboptimal adherence to insulin treatment is a main issue in adolescents with type 1 diabetes. However, to date, there are no available data on adherence to adjunct non-insulin medications in this population. Our aim was to assess adherence to ACE inhibitors and statins and explore potential determinants in adolescents with type 1 diabetes.
\end{abstract}

Research Design and Methods-443 adolescents with type 1 diabetes were recruited into the Adolescent Type 1 Diabetes cardio-renal Intervention Trial (AdDIT) trial and exposed to treatment with two oral drugs: an ACE inhibitor, a statin, combinations of both or placebo for 2-4 years. Adherence was assessed every 3 months with the Medication Event Monitoring System (MEMS) and pill count.

\footnotetext{
Corresponding author: Dr M. Loredana Marcovecchio; MD, PhD, University Department of Paediatrics, Level 8, Box 116, Addenbrooke's Hospital, Hills Road, Cambridge CB2 0QQ, UK, Tel.: +44 (0) 1223 768615, Fax: +44 (0) 1223 336996, mlm45@medschl.cam.ac.uk.

Author contributions

Dr Marcovecchio has full access to the data and takes responsibility for the integrity of the data and the accuracy of the data analysis. EN was involved in the literature review, data interpretation and analysis and wrote the first draft of the manuscript. CLA contributed to data acquisition and interpretation and critical review of the manuscript. STC and TS contributed to data acquisition and analysis, critical review of the manuscript. RND, DD, JED, TWJ, FHM, SMM, HAWN, DBD, contributed to the study concept and design, data interpretation and critical review of the manuscript. MLM contributed to study design and concept, data interpretation, performed statistical analysis, wrote and reviewed the manuscript.

Conflict of interest

None in related to the present work.
} 
Results-Median adherence during the trial was $80.2 \%$ (interquartile range: 63.6-91.8), based on MEMS, and 85.7\% (72.4-92.9) for pill count. MEMS- and pill count-based adherence dropped from $92.9 \%$ and $96.3 \%$, respectively, at the first visit, to $76.3 \%$ and $79.0 \%$ at the end of the trial. The percentage of study participants with adherence $\geq 75 \%$ declined from $84 \%$ to $53 \%$. A good correlation was found between MEMS- and pill count-based adherence $(r=0.82, p<0.001)$. Factors associated with adherence were age, glycemic control and country.

Conclusions-We report an overall good adherence to ACE inhibitors and statins during a clinical trial, although there was a clear decline in adherence over time. Older age and suboptimal glycemic control at baseline predicted lower adherence during the trial, and predictably reduced adherence was more prevalent in subjects who subsequently dropped out.

Adherence to a medication regimen, defined as the extent to which a person's medicationtaking behavior corresponds with the agreed recommendations from a healthcare provider, is a key determinant of response to therapy and patients' outcomes (1). Adherence is a complex and multifactorial paradigm, which varies depending on the disease and treatment regimen, and is also influenced by physician and patient-related factors (1-3).

In adolescents with type 1 diabetes, suboptimal adherence to diabetes self-management, including insulin therapy, is a main concern $(4,5)$, and it reflects age-related issues as well as the complexity of diabetes management $(6,7)$. Insulin is the standard treatment for type 1 diabetes, but its doses and timing of administration need to be coordinated, on a daily basis, with the results of blood glucose monitoring, dietary intake, levels of physical activity as well as potential intercurrent illnesses and other factors (6). The burden of adhering to these various behaviors is carried by patients and their families and can affect every aspect of daily life.

Given that glycemic control is often suboptimal during adolescence (8), there is a growing interest in implementing new non-insulin adjunct drug therapies to achieve recommended glycemic targets, and to prevent short- and long-term complications (9). International guidelines are also more widely recommending treatments with blood pressure and lipid lowering drugs during adolescence in the presence of cardiovascular risk factors such as dyslipidemia and hypertension $(10,11)$. Understanding how these adjunct medications will be accepted and adhered to by young people is needed, not least to inform potential strategies to improve and maintain treatment adherence following their implementation into clinical practice. Although there is clear evidence for a strong association between adherence to diabetes treatment and glycemic control and, in turn, complication risk (12), there are no available data on adherence to adjunct non-insulin medications in adolescents with type 1 diabetes.

The Adolescent Type 1 Diabetes cardio-renal Intervention Trial (AdDIT) recruited a contemporary cohort of adolescents with type 1 diabetes from three countries (UK, Canada and Australia) (13). Trial participants were requested to take two oral medications, angiotensin-converting enzyme (ACE) inhibitors and statins, or related placebos, daily for a duration of 2-4 years. Thus, this trial offers a unique opportunity to look at adherence to medications other than standard insulin treatment. As part of the trial, adherence was assessed with the Medication Event Monitoring System (MEMS), which records the date 
and time of each opening of a pill container, and is currently considered the gold standard for tracking drug dosing history in clinical trials $(14,15)$. Pill count was used as a secondary indirect method to assess adherence. Using data from the AdDIT trial we aimed: 1) to assess rates of adherence and its changes over time; 2) to compare MEMS and pill count as methods of assessing adherence, and 3) to assess factors predicting adherence, in adolescents with type 1 diabetes in the context of a clinical trial.

\section{Methods}

\section{Study population}

The study cohort included 443 adolescents with type 1 diabetes, aged 10-16 years, recruited into the AdDIT trial. The design and results of the AdDIT trial have been previously reported (13). In brief, AdDIT was a multicenter, double-blind, randomized, placebocontrolled trial conducted at 32 centers across the UK, Canada and Australia. AdDIT was designed to explore the potential cardio-renal protection provided by ACE inhibitors and statins in adolescents with type 1 diabetes at increased risk of vascular complications based on an albumin-creatinine ratio (ACR) in the upper tertile of the normal range (13).

The inclusion criteria for the AdDIT trial were: age between 10 and 16 years; a duration of diabetes of at least 1 year (or a diagnosis within the past year with an undetectable C-peptide level); an adjusted ACR in the upper tertile of the screened population. Exclusion criteria were non-type 1 diabetes, pregnancy or unwillingness to adhere to contraceptive advice and pregnancy testing, severe hyperlipidemia or a family history of familial hypercholesterolemia, hypertension unrelated to diabetic nephropathy, previous exposure to the investigational drugs, unwillingness or inability to adhere to the trial protocol, the presence of coexisting conditions (excluding treated hypothyroidism and celiac disease), proliferative retinopathy, and the presence of renal disease that was not associated with type 1 diabetes (13). Participants were randomized, using a 2-by-2 factorial design, to receive a variable dose of an ACE inhibitor (Quinapril, 5 or $10 \mathrm{mg}$ ), a fixed dose of a statin (Atorvastatin, $10 \mathrm{mg}$ ), combinations of both drugs or matched placebos. The drugs were given orally as two tablets daily for a duration of minimum 2 up to maximum 4 years. Trial duration varied based on the time when each participant was enrolled, during the recruitment timescale.

The trial conformed to the provisions of the Declaration of Helsinki and was approved by the Cambridge University Hospitals and participating local research ethics committees. Parents of the participants provided written informed consent, and the trial participants were asked to provide their written assent if they were not yet at an age when they could provide consent.

\section{Assessment of adherence}

Electronic monitoring: Quinapril and Atorvastatin and the matched placebos were supplied at each study visit. The tablets were provided in containers with the electronic monitoring caps MEMS (provided by AARDEX, Zug, Switzerland), that recorded the precise date and time of each opening and closing in order to track adherence (13). Each study participant 
was provided with two bottles containing the study medications/placebos. Participants were asked to keep the tablets in the bottles provided and not to transfer them to other containers. They were informed that their adherence to both drugs was monitored during the trial, and at each study visits they were encouraged to take the medications. However, they did not receive any incentives to promote adherence and no detailed data on adherence to diabetes self-management were collected during the trial.

At each study visit, data from the caps were read by MEMS devices and stored. MEMS adherence was estimated assuming that each cap opening represented a participant taking the correct number of medications from the container during the study period. Participants who did not open their MEMS cap were rated as non-adherent for that day.

Pill count: At every study visit, unused tablets were brought back and counted. By both methods, adherence was assessed at 1 month after randomization and then every three months. It was calculated as the percentage of days on which a tablet was apparently taken by participants remaining active in the study, out of the days in between study visits.

\section{Predictors of adherence}

Potential predictors of adherence were chosen 'a priori' from participants' characteristics assessed at baseline and included in the randomization process (13). These included: chronological age, sex, diabetes duration, age at diabetes diagnosis, baseline glycemic control as assessed by glycated hemoglobin (HbA1c), method of diabetes treatment (multiple daily injections (MDI) or continuous subcutaneous insulin infusion (CSII)), country (UK, Australia, Canada).

\section{Statistical analysis}

Results are presented as percentages, median, and interquartile ranges (IQR). Linear mixed model was used to compare adherence rate across the study visits. Mann-Whitney and Kruskall-Wallis tests were used to compare differences in overall adherence between study groups. Linear regression models were used to assess factors associated with overall adherence. Bland-Altman plots were used to assess agreement between the MEMS and pill count methods. Two-sided p-values less than 0.05 were considered statistically significant. Analyses were performed with SPSS, version 25.

\section{Results}

\section{Baseline characteristics of the study population}

443 adolescents (203 females and 240 males; 376 white and 67 from other ethnic groups) were randomized into the AdDIT trial at a median age of 13.8 (IQR 12.6-15.0) years and median diabetes duration of 5.0 (3.2-7.8) years across three countries: Australia $(\mathrm{n}=201$, $45 \%)$, Canada ( $\mathrm{n}=124,28 \%$, UK $(\mathrm{n}=118,27 \%)$. Their baseline median (IQR) HbA1c was $8.3(7.6-9.3) \%$ [67.2 (59.6-78.1) $\mathrm{mmol} / \mathrm{mol}] ; 266(60 \%)$ were on MDI and $177(40 \%)$ on CSII. 
These 443 study participants represented around $43 \%$ of the eligible screened population and their demographic characteristics were similar to those who refused to take part to the study (data not shown).

Study participants were followed for a median of 2.6 years and their adherence was assessed starting from 1 month after randomization and every 3 months thereafter, until completion of the trial after 2-4 years.

\section{Adherence rate based on MEMS vs pill count}

There were no significant differences between adherence to ACE Inhibitor or related placebo and statins or related placebo (Supplementary Figure S1). The average adherence for both drugs was used in all analyses. Levels of adherence between the active drugs vs placeboplacebo groups during the whole trial period were similar (81.6 (66.5-92.2)\% vs 79.9 $(62.9-91.7) \%), \mathrm{p}=0.54$.

Overall median adherence (IQR) was $80.2 \%$ (63.6-91.8) [mean \pm SD: $75.0 \pm 20.8 \%$ ], based on MEMS, and $85.7 \%$ (72.4-92.9) [mean \pm SD: $80.4 \pm 17.0 \%$ ], based on pill count. Adherence based on both MEMS and pill count dropped from $92.9 \%$ and $96.3 \%$, respectively, at the first visit, to $76.3 \%$ and $79.0 \%$ at the last visit ( $\mathrm{p}$ for trend $<0.001$ ). As shown in Figure 1, the main decline in adherence occurred during the first 18 months (adherence at 18 months: MEMS $=79.8 \%$; Pill count $=83.2 \%$ ) ( $p$ for trend $<0.001)$, and after that it remained almost stable (Figure 1). The sample size, as reported in Figure 1, decreased over time, mainly due to participants completing the minimum duration of 2 years in the trial. In addition, there were 78 participants who dropped out at different time points and 37 participants who stopped treatment earlier than expected but remained in the study. Adherence during the trial was lower in participants who subsequently dropped out or stopped medications compared to the rest of the trial population remaining active in the trial $(72.4(53.2-90.3) \%$ vs 82.0 (66.2 -92.2)\%), $\mathrm{p}=0.02$. When including these non-active participants, with their adherence considered equal to 0 in the analysis, overall adherence during the trial was $4.4 \%$ lower compared to the adherence calculated only for participants remaining in the study (Supplementary Figure S2).

The correlation between MEMS- and pill count-based adherence was $0.82(\mathrm{p}<0.001)$ (Figure 2a). Bland Altman plots showed good agreement between the two methods with a bias of 5.3\% (95\% confidence interval: -28.2; 17.9) (Figure 2b).

\section{Levels of adherence over study treatment visits based on MEMS data}

When analyzing the data using different categories of adherence ( $\geq 75 \%, 50-75 \%, 25-50 \%$ and $<25 \%$ ), most participants had a median adherence rate above $75 \%$ during the trial period (Figure 3). However, the percentage of active participants with adherence $\geq 75 \%$ decreased from $84 \%$ at the beginning of the study to $56-58 \%$ after $18-24$ months and to $53 \%$ after 48 months. In parallel the proportion of participants with adherence rates between $50 \%$ and $75 \%, 50 \%$ and $25 \%$ and less than $25 \%$ increased from $13 \%$ to $28 \%, 1.8 \%$ to $14 \%$ and $0.7 \%$ to $4.6 \%$, respectively. 


\section{Baseline factors associated with adherence based on MEMS data}

Adherence decreased across age groups (10-12, 12-15 and 15-17 year-old group): 81.4 (70.1-93.4)\% vs 79.3 (60.8-91.0)\% vs 78.9 (60.8-91.7)\%, although the between-group difference reached only borderline statistical significance $(\mathrm{p}=0.07)$.

No differences in adherence during the study period were found between participants with a diabetes duration, at the baseline visit, less than 5 years (80.6 (65.7-91.8)\%) compared to those with a duration of 5-10 years $(80.4(66.8-91.8) \%), \mathrm{p}=0.56$. No differences were found between males (79.8 (63.4-91.0)\%) and females (80.4 (64.3-92.6)\%), $\mathrm{p}=0.63$.

When comparing adherence between the three countries involved in the AdDIT trial, Australia showed a slightly higher median adherence (83.4 (70.1-92.9)\%) compared to the UK (78.9 (61.7-91.6)\%) and Canada (73.8 (56.8-88.3)\%), p for trend=0.001. However, the decline in adherence during the trial period was observed in all three countries: at the first study visit, median adherence was $93.3 \%$ for Australia, $92.9 \%$ for the UK and $89.0 \%$ for Canada, whereas by the last study visit it dropped to $79.8 \%, 76.1$ and $73.0 \%$, respectively.

Levels of adherence varied in relation to glycemic control at the baseline study visit, with the lowest adherence in participants with an $\mathrm{HbAlc}>8.5 \%$ ( $>69 \mathrm{mmol} / \mathrm{mol}$ ): 76.9 (56.9-88.8)\%, compared to those with an $\mathrm{HbAlc}$ between 7.5 and $8.5 \%(58-69 \mathrm{mmol} / \mathrm{mol})$ : $81.9(66.5-91.7) \%$ and less than $7.5 \%(<58 \mathrm{mmol} / \mathrm{mol}): 88.1$ [75.2-93.9]\%, $\mathrm{p}=0.001$ (Supplementary Table 1 and Supplementary Figure S3). There were no significant differences in adherence between participants on MDI and those on CSII: 80.2 (60.6-92.2)\% vs $80.5(67.6-91.9) \%$, $\mathrm{p}=0.84$.

In univariate linear regression models, baseline factors significantly associated with adherence were chronological age, with a lower adherence in older study participants; country of origin, with the highest adherence in Australia; glycemic control, with the lowest adherence associated with the highest HbA1c levels (Table 1). In a multiple regression model, $\mathrm{HbAlc}$ and country remained independently associated with adherence (Table 1).

\section{Discussion}

This is the major study providing data on adherence to medications other than insulin in a large contemporary cohort of adolescents with type 1 diabetes, recruited across three countries, and enrolled in a clinical trial involving taking two adjunct oral medications daily, for a total duration of 2-4 years.

Median adherence to both ACE inhibitors and statins during the AdDIT trial was around $80 \%$, although there was a deterioration in adherence over time. Adherence to medications in adolescents, in clinical practice and in clinical trials, varies widely from 10 to $90 \%$, with most studies reporting rates less than 50\%, particularly in those with chronic rather than acute conditions (16). The physical and psychological changes occurring during adolescence, along with demographic and socioeconomic factors and the intrinsic characteristics of the underlying disease, can all contribute to poor adherence in this age group (17). 
Type 1 diabetes is a good example of a chronic disease where adherence to treatment strategies and self-management can be challenging, particularly during adolescence (18). Current diabetes self-management is based on MDI or CSII, which need to be balanced with frequent blood glucose monitoring, diet, physical activity as well as other factors, such as intercurrent illnesses (18). Observational studies based on self-reports, questionnaires, $\mathrm{HbA1c}$ and, more recently, on continuous glucose monitoring and insulin pumps, indicate suboptimal adherence to insulin treatment and other self-management behaviors among children and adolescents with type 1 diabetes (19-24). In one of these studies, adherence to individual components of treatment regimens varied from $29 \%$ for dietary recommendations to $52 \%$ for insulin administration and $69 \%$ for blood glucose monitoring (24). In addition, around $65 \%$ of insulin pump-wearing children miss one or more meal time boluses of insulin per week (20). These data are a concern given that non-adherence to diabetes regimen can result in suboptimal glycemic control and increased episodes of diabetic ketoacidosis and, in the long term, lead to higher rates of vascular complications and reduced life expectancy (25).

In contrast to these real life data, AdDIT provides valuable information to adherence to adjunct oral medications in the context of a clinical trial. Although, non-adherence to ACE inhibitors or statins does not have the same impact of non-adherence to insulin in terms of risk for acute complications, understanding factors affecting adherence to these medications is important to maximize their benefit when used in clinical practice. This is important given that use of these drugs from an early age may improve long-term outcomes of young people with type 1 diabetes.

Although overall adherence was better than expected, during the AdDIT trial, there was a deterioration in adherence to both ACE Inhibitors and statins over time, and after 2 years of treatment $58 \%$ of participants showed an adherence of at least $75 \%$ and this percentage dropped to $53 \%$ after 4 years. This is in line with previous findings from other studies, and likely reflect loss of participants' motivation, feeling of lack of immediate benefits, as well as the age-related factors $(5,17,26)$. Overall, these data highlight the need of strategies to reinforce adherence over time to gain the maximum benefit from any intervention during a clinical trial and in the real word setting.

The AdDIT trial provides valuable data on how adherence can vary based on the methods of assessment. Several methods can be used to assess adherence, and they are broadly classified as direct and indirect methods (27). Direct methods, based on the measurement of the level of the administered drug or its metabolites in biological samples, are the most accurate, but also most expensive and impractical ways to assess adherence. In contrast, indirect methods rely on patient self-reports or questionnaires, rates of prescription refills, pill counts and are considered less precise and more prone to errors $(1,28)$. Pill count is one of the most used indirect methods to assess drug adherence but it can be susceptible to misrepresentation and overestimate patient's adherence $(27,29)$. The MEMS method, which records the date and time of each opening of a pill container, is considered a more accurate indirect method and the gold standard for tracking drug dosing history in clinical trials $(14,15)$. MEMS has been used to investigate adherence in adult populations with various conditions (30), but only in a few clinical trials involving pediatric populations. These were mainly studies with small 
sample sizes and short periods of exposures to medications, ranging from 1 up to 12 months (31-33).

In the AdDIT population two indirect methods were used, namely pill count and MEMS, and this comparison confirmed that pill count tends to overestimate adherence, as previously reported $(27,29)$. This overestimation with pill count occurred even though the study participants were aware of their adherence being monitored with the MEMS caps. Reasons for this attempt to mislead about adherence may include inadequate knowledge about the aim or benefits of treatment as well as dissatisfaction with treatment (1).

The AdDIT trial also offered the opportunity to assess potential factors associated with adherence in the context of a clinical trial. Adherence to a medical regimen can be affected by caregiver-related factors, patients characteristics, relationships between healthcare professionals and patients, social and cultural circumstances and disease- and treatmentrelated factors $(2,3)$. Frequently, patients are able to memorise only $50 \%$ of discussed issues during clinical visits (34) and may have insufficient knowledge about drug usage, and that could lead to patients not taking the appropriate prescribed doses, missing doses or discontinuing treatment (1). Another potential main barrier to adherence to prescribed drugs are disbelief related to the diagnosis, fear of side effects, high frequency of dosing, number of concurrent medications, routes of drug administration and long-term treatment (3).

Medication adherence in children and adolescents is generally more complex than in adults, and this is reflected by worse adherence rates reported in pediatric than in adult studies (35). Younger children require involvement of a third party in the management of their medical condition (17), while adolescents face a particular life phase, characterized by many challenges potentially conflicting with optimal adherence (36).

In the AdDIT trial, adherence was lower in older than younger participants, in line with the results of previous studies suggesting a decline in adherence during the transition from childhood to adolescence (37). This may reflect specific developmental changes occurring during adolescence and less parental involvement in diabetes management (38), which is a known step as children grow up and express their desire of being more independent.

Surprisingly, we did not find any effect of diabetes duration on adherence rates, in contrast to other studies reporting a decline with longer disease duration (37). Our findings could be explained by the relatively short and narrow range of diabetes duration in our study population.

Despite the implementation of a standardized protocol in the AdDIT trial, there were differences in adherence across countries, with a slightly higher adherence to therapy in Australia than in the UK and Canada. Variations in adherence between countries have been previously reported and could be related to socio-demographic and socio-economic factors, differences in health care systems and medical care, and physician-patient communication $(1,2,39)$.

Suboptimal glycemic control was also associated with lower adherence to the adjunct oral medications during the AdDIT trial, likely reflecting participants' specific characteristics 
and behaviors which affect not only adherence to insulin regimes but also to any additional treatment strategies. Adolescents with type 1 diabetes face several obstacles to treatment adherence, including psychosocial issues, model of family functioning, communication, and regimen-associated barriers (36). They are also at high risk of depression, anxiety, other mood or eating disorders (18). All these factors have previously been associated with poor adherence to insulin treatment (36), and may similarly impact adherence to adjunct oral medications.

The association between suboptimal glycemic control and lower adherence across all randomized groups during the AdDIT trial raises the question as to whether more complex treatment approaches, which should be directed mainly towards those patients not achieving recommended glycemic targets, could bring real benefits. Currently, several adjunct noninsulin treatments are being investigated with the aim of improving glycemic control in people with type 1 diabetes, as well as addressing weight control, preserving beta cell function and inducing vascular protection (9-11). These include use of gliptin and sodiumglucose co-transporters 2 inhibitor agents, as well as other drugs to reduce cardiovascular risk factors, such as statins and ACE inhibitors. However, before the implementation of more complex drug therapies, strategies examining and overcoming the issues of poor adherence in those adolescents already non-adhering to current insulin treatment regimen are required (3).

Some limitations of the present study need to be acknowledged. Although the study population was demographically well representative of the larger multinational AdDIT screening population of over 4000 adolescents with type 1 diabetes, the adherence data related to a selective group who agreed to participate in a clinical trial and were aware that adherence to the adjunct medications was monitored. This likely resulted in a greater adherence than that observed in daily clinical practice, where there is no specific standardized monitoring system in place, and thus the findings are less generalizable to a real-word setting. Participants remained active in the study for a variable duration, from a minimum of 2 to a maximum of 4 years, leading to adherence data available only for around $30 \%$ of the study population for the whole 4-year trial duration. Although the MEMS method seems superior to pill count or other indirect methods, it could still have disadvantages such as misleading the system when opening the container without taking the drug or taking the wrong number of tablets or multiple doses out of the container at the same time (27). In addition, we did not collect data on socioeconomic status or psychosocial factors (family, peers, social support, acceptance/understanding of disease and treatment, mental status, self-esteem), which have previously been reported to affect adherence in children and adolescents in some, although not all studies $(26,40)$. Finally, we were unable to assess the effect of other potential predictors of adherence, such as ethnicity (40), given that the participants were predominantly White.

\section{Conclusions}

Overall, AdDIT has provided valuable insights into medication adherence in the context of multiple drug treatment for young people with T1D. The AdDIT trial showed an overall good adherence rate in a population of adolescents with type 1 diabetes in a clinical trial 
setting, but also confirmed that deterioration of adherence over time can be an issue. Older age and higher $\mathrm{HbAlc}$ at baseline predicted adolescents with lower adherence during the trial, highlighting two targets for strategies aiming at improving adherence both in clinical trials and in daily clinical practice. Although type 1 diabetes is a complex condition, based on the present data the implementation of adjunct oral therapies in type 1 diabetes in the future seems feasible, but will require specific strategies addressing potential barriers to adherence and ways of overcoming them.

\section{Supplementary Material}

Refer to Web version on PubMed Central for supplementary material.

\section{Funding/Support}

AdDIT was funded by Diabetes UK, Juvenile Diabetes Research Foundation, the British Heart Foundation and in Canada the JDRF- Canadian Clinical Trial Network (CCTN), the Canadian Diabetes Association and the Heart and Stroke Foundation Canada. The study funders had no role in the design and conduct of the study; collection, management, analysis, and interpretation of the data; and preparation E. Niechcial was supported by a European Foundation for the Study of Diabetes (EFSD) Albert Renold Travel Fellowship Programme We also acknowledge support from the National Institute for Health Research (NIHR) Cambridge Biomedical Research Centre, the NIHR Cambridge Clinical Trials Unit and the UK NIHR Clinical Research Network. We thank all participants for their involvement and commitment.

\section{References}

1. Osterberg L, Blaschke T. Adherence to Medication. N Engl J Med. 2005; 353:487-97. [PubMed: 16079372]

2. Dawson LA. What factors affect adherence to medicines? Arch Dis Child - Educ Pract Ed. 2018; 2017

3. Kini V, Ho PM. Interventions to Improve Medication Adherence. JAMA. 2018; 320:2461. [PubMed: 30561486]

4. Hauser ST, Jacobson AM, Lavori P, Wolfsdorf JI, Herskowitz RD, Milley JE, et al. Adherence among children and adolescents with insulin-dependent diabetes mellitus over a four-year longitudinal follow-up: II. Immediate and long-term linkages with the family milieu. J Pediatr Psychol. 1990; 15:527-42. [PubMed: 2258799]

5. Alan MJ, Hauser ST, Lavori P, Wolfsdorf JI, Herskowitz RD, Milley JE, et al. Adherence among children and adolescents with insulin-dependent diabetes mellitus over a four-year longitudinal foliow-up: I. The influence of patient coping and adjustment. J Pediatr Psychol. 1990; 15:511-26. [PubMed: 2258798]

6. Chiang JL, Maahs DM, Garvey KC, Hood KK, Laffel LM, Weinzimer SA, et al. Type 1 Diabetes in Children and Adolescents: A Position Statement by the American Diabetes Association. Diabetes Care. 2018; 41:2026-44. [PubMed: 30093549]

7. Farsaei S, Radfar M, Heydari Z, Abbasi F, Qorbani M. Insulin adherence in patients with diabetes: Risk factors for injection omission. Prim Care Diabetes. 2014; 8:338-45. [PubMed: 24721139]

8. Miller KM, Foster NC, Beck RW, Bergenstal RM, DuBose SN, DiMeglio LA, et al. Current state of type 1 diabetes treatment in the U.S.: updated data from the T1D Exchange clinic registry. Diabetes Care. 2015; 38:971-8. [PubMed: 25998289]

9. Naciu AM, Pozzilli P. Novel blood glucose lowering therapies for managing type 1 diabetes in paediatric patients. Expert Opin Pharmacother. 2018; 19:355-64. [PubMed: 29460641]

10. Donaghue KC, Marcovecchio ML, Wadwa RP, Chew EY, Wong TY, Calliari LE, et al. ISPAD Clinical Practice Consensus Guidelines 2018: Microvascular and macrovascular complications in children and adolescents. Pediatr Diabetes. 2018; 19:262-74. [PubMed: 30079595] 
11. American Diabetes Association. 12. Children and Adolescents:Standards of Medical Care in Diabetes-2018. Diabetes Care. 2018; 41:S126-36. [PubMed: 29222383]

12. Hood KK, Peterson CM, Rohan JM, Drotar D. Association Between Adherence and Glycemic Control in Pediatric Type 1 Diabetes: A Meta-analysis. Pediatrics. 2009; 124:e1171-9. [PubMed: 19884476]

13. Marcovecchio ML, Chiesa ST, Bond S, Daneman D, Dawson S, Donaghue KC, et al. ACE Inhibitors and Statins in Adolescents with Type 1 Diabetes. N Engl J Med. 2017; 377:1733-45. [PubMed: 29091568]

14. Svarstad BL, Chewning BA, Sleath BL, Claesson C. The brief medication questionnaire: A tool for screening patient adherence and barriers to adherence. Patient Educ Couns. 1999; 37:113-24. [PubMed: 14528539]

15. Vrijens B, Urquhart J. Methods for measuring, enhancing, and accounting for medication adherence in clinical trials. Clin Pharmacol Ther. 2014; 95:617-26. [PubMed: 24739446]

16. Rapoff, M. Adherence to pediatric medical regimens. New York, NY: Kluwer Academic/Plenum Publishers; 1999.

17. Matsui DM. Drug compliance in pediatrics: Clinical and research issues. Pediatr Clin North Am. 1997; 44:1-14. [PubMed: 9057780]

18. Cameron FJ, Garvey K, Hood KK, Acerini CL, Codner E. ISPAD Clinical Practice Consensus Guidelines 2018: Diabetes in adolescence. Pediatr Diabetes. 2018; 19:250-61. [PubMed: 29900653]

19. Hsin O, La Greca AM, Valenzuela J, Moine CT, Delamater A. Adherence and glycemic control among hispanic youth with type 1 diabetes: Role of family involvement and acculturation. J Pediatr Psychol. 2010; 35:156-66. [PubMed: 19491214]

20. Burdick J, Chase HP, Slover RH, Knievel K, Scrimgeour L, Maniatis AK, et al. Missed Insulin Meal Boluses and Elevated Hemoglobin A1c Levels in Children Receiving Insulin Pump Therapy. Pediatrics. 2004; 113:e221-4. [PubMed: 14993580]

21. Driscoll KA, Young-Hyman D. Use of Technology When Assessing Adherence to Diabetes SelfManagement Behaviors. Curr Diab Rep. 2014; 14:521. [PubMed: 25023214]

22. Westen SC, Warnick JL, Albanese-O’Neill A, Schatz DA, Haller MJ, Entessari M, et al. Objectively Measured Adherence in Adolescents With Type 1 Diabetes on Multiple Daily Injections and Insulin Pump Therapy. J Pediatr Psychol. 2019; 44:21-31. [PubMed: 30184209]

23. Patton SR, Driscoll KA, Clements MA. Adherence to Insulin Pump Behaviors in Young Children With Type 1 Diabetes Mellitus. J Diabetes Sci Technol. 2017; 11:87-91. [PubMed: 27390223]

24. Driscoll KA, Johnson SB, Hogan J, Gill E, Wright N, Deeb LC. Insulin Bolusing Software: The Potential to Optimize Health Outcomes in Type 1 Diabetes Mellitus. J Diabetes Sci Technol. 2013; 7:646-52. [PubMed: 23759397]

25. Fortin K, Pries E, Kwon S. Missed Medical Appointments and Disease Control in Children With Type 1 Diabetes. J Pediatr Heal Care. 2016; 30:381-9.

26. Lam WY, Fresco P. Medication Adherence Measures: An Overview. Biomed Res Int. 2015; 2015:217047. [PubMed: 26539470]

27. Lee CH, Hui EYL, Woo YC, Yeung CY, Chow WS, Yuen MMA, et al. Circulating fibroblast growth factor 21 levels predict progressive kidney disease in subjects with type 2 diabetes and normoalbuminuria. J Clin Endocrinol Metab. 2015; 100:1368-75. [PubMed: 25625802]

28. Lehmann A, Aslani P, Ahmed R, Celio J, Gauchet A, Bedouch P, et al. Assessing medication adherence: Options to consider. Int J Clin Pharm. 2014; 36:55-69. [PubMed: 24166659]

29. El Alili M, Vrijens B, Demonceau J, Evers SM, Hiligsmann M. A scoping review of studies comparing the medication event monitoring system (MEMS) with alternative methods for measuring medication adherence. Br J Clin Pharmacol. 2016; 82:268-79. [PubMed: 27005306]

30. McGrady ME, Williams SN, Davies SM, Pai ALH. Adherence to outpatient oral medication regimens in adolescent hematopoietic stem cell transplant recipients. Eur J Oncol Nurs. 2014; 18:140-4. [PubMed: 24361039]

31. Aylward BS, Rausch JR, Modi AC. An examination of 1-year adherence and persistence rates to antiepileptic medication in children with newly diagnosed epilepsy. J Pediatr Psychol. 2015; 40:66-74. [PubMed: 24648257] 
32. Hommel KA, Davis CM, Baldassano RN. Objective versus subjective assessment of oral medication adherence in pediatric inflammatory bowel disease. Inflamm Bowel Dis. 2009; 15:589-93. [PubMed: 18985746]

33. Kessels RPC. Patients' memory for medical information. J R Soc Med. 2003; 96:219. [PubMed: 12724430]

34. Staples B, Bravender T. Drug compliance in adolescents: Assessing and managing modifiable risk factors. Pediatric Drugs. 2002; 4:503-13. [PubMed: 12126454]

35. Borus JS, Laffel L. Adherence challenges in the management of type 1 diabetes in adolescents: prevention and intervention. Curr Opin Pediatr. 2010; 22:405-11. [PubMed: 20489639]

36. Hilliard ME, Wu YP, Rausch J, Dolan LM, Hood KK. Predictors of Deteriorations in Diabetes Management and Control in Adolescents With Type 1 Diabetes. J Adolesc Heal. 2013; 52:28-34.

37. Berg CA, Butler JM, Osborn P, King G, Palmer DL, Butner J, et al. Role of parental monitoring in understanding the benefits of parental acceptance on adolescent adh erence and metabolic control of type 1 diabetes. Diabetes Care. 2008; 31:678-83. [PubMed: 18202244]

38. Larsen J, Stovring H, Kragstrup J, Hansen DG. Can differences in medical drug compliance between European countries be explained by social factors: analyses based on data from the European Social Survey, round 2. BMC Public Health. 2009; 9:145. [PubMed: 19445714]

39. Gallegos-Macias AR, Macias SR, Kaufman E, Skipper B, Kalishman N. Relationship between glycemic control, ethnicity and socioeconomic status in Hispanic and white non-Hispanic youths with type 1 diabetes mellitus. Pediatr Diabetes. 2003; 4:19-23. [PubMed: 14655519]

40. Katz LL, Anderson BJ, McKay SV, Izquierdo R, Casey TL, Higgins LA, et al. Correlates of medication adherence in the TODAY cohort of youth with type 2 diabetes. Diabetes Care. 2016; 39:1956-62. [PubMed: 27352955] 


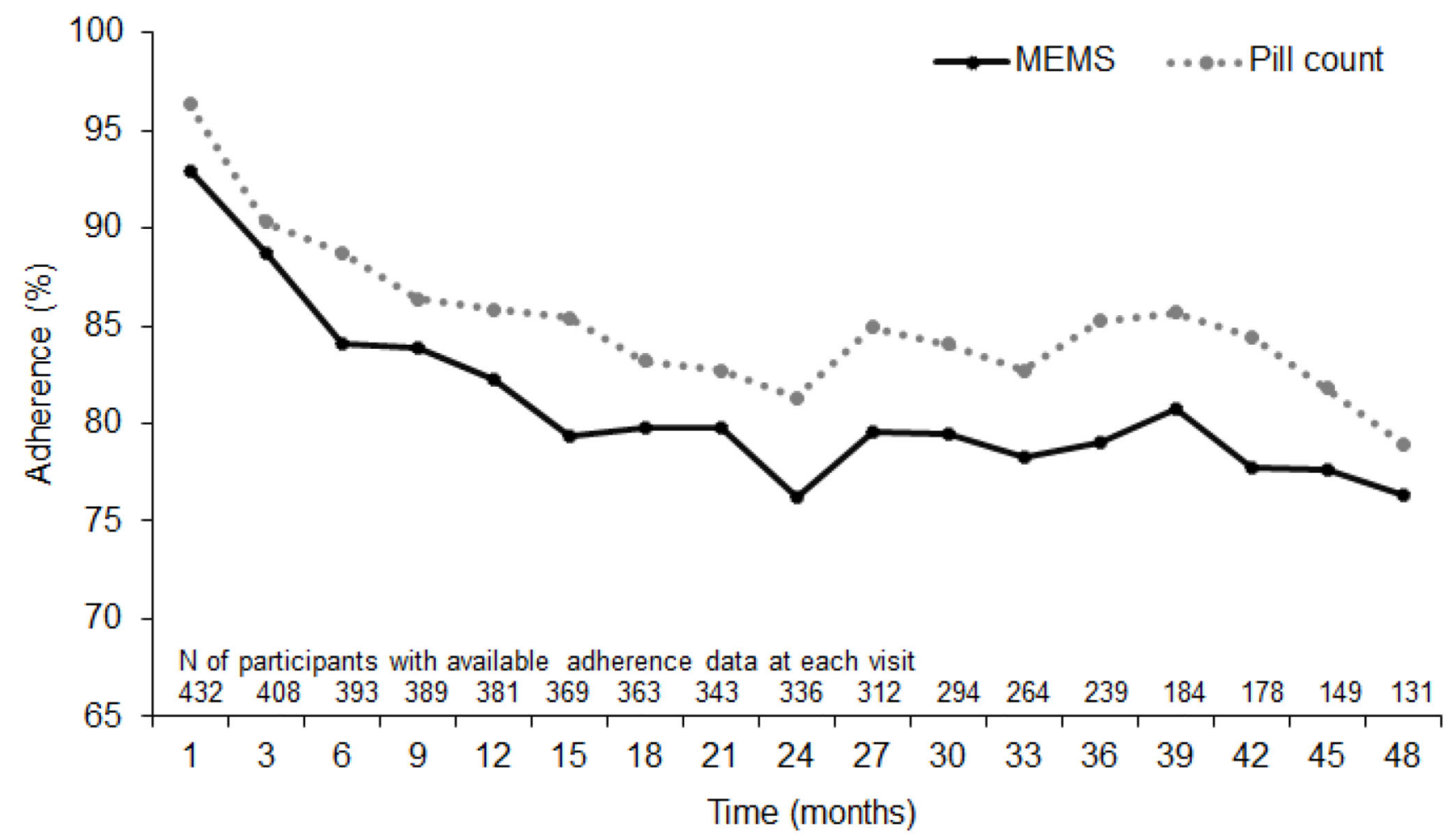

Figure 1.

Adherence during the trial period based on MEMS and pill count

Results at each study visit are reported as medians. N of participants are those still active at each follow up study visit with available adherence data. At the first 1-month visit. P for over time changes in each group: $<0.001$ 
a

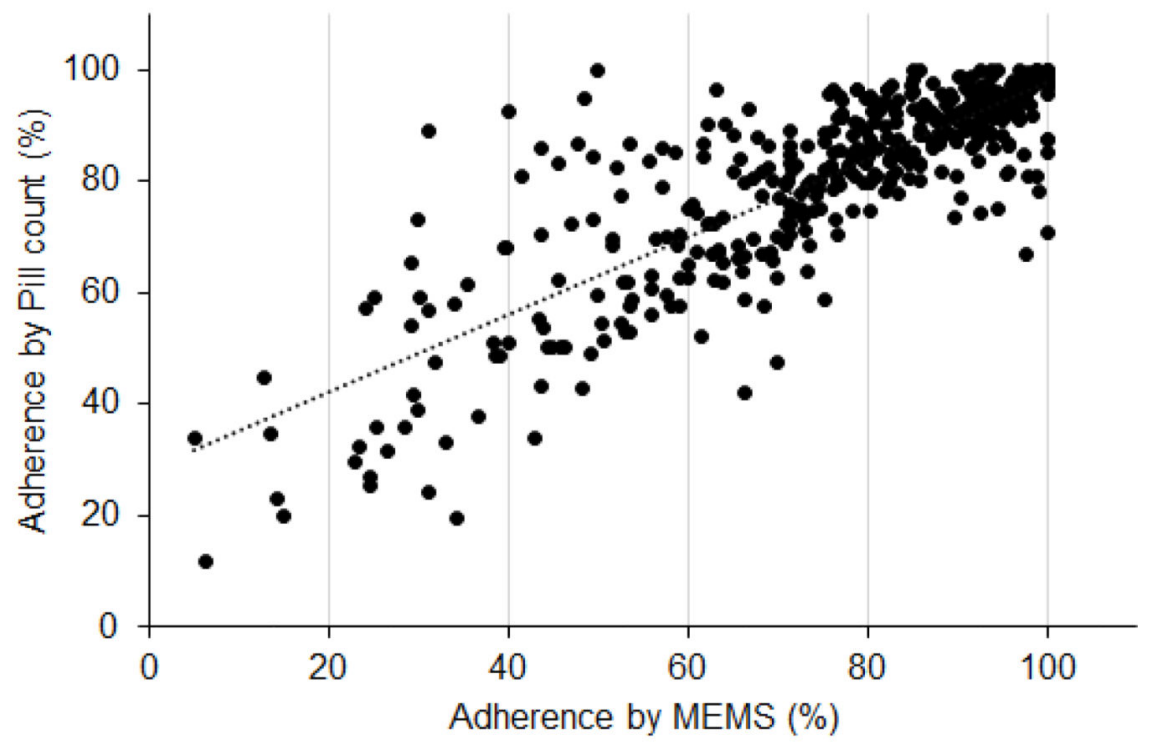

b

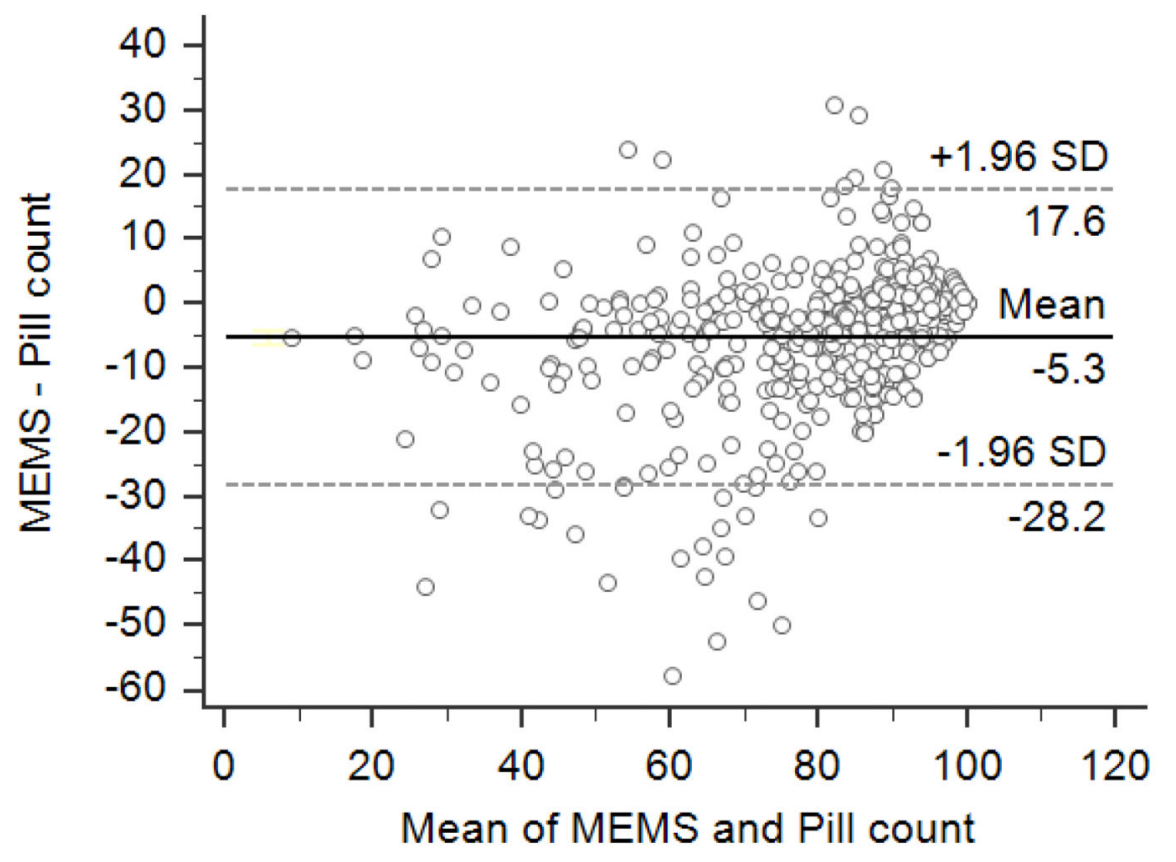

Figure 2.

Comparisons between adherence assessed by MEMS and pill count: A) Scatter plot of pill count vs MEMS, $r=0.82$, $p<0.001$. B) Bland-Altman plots of the two adherence methods 


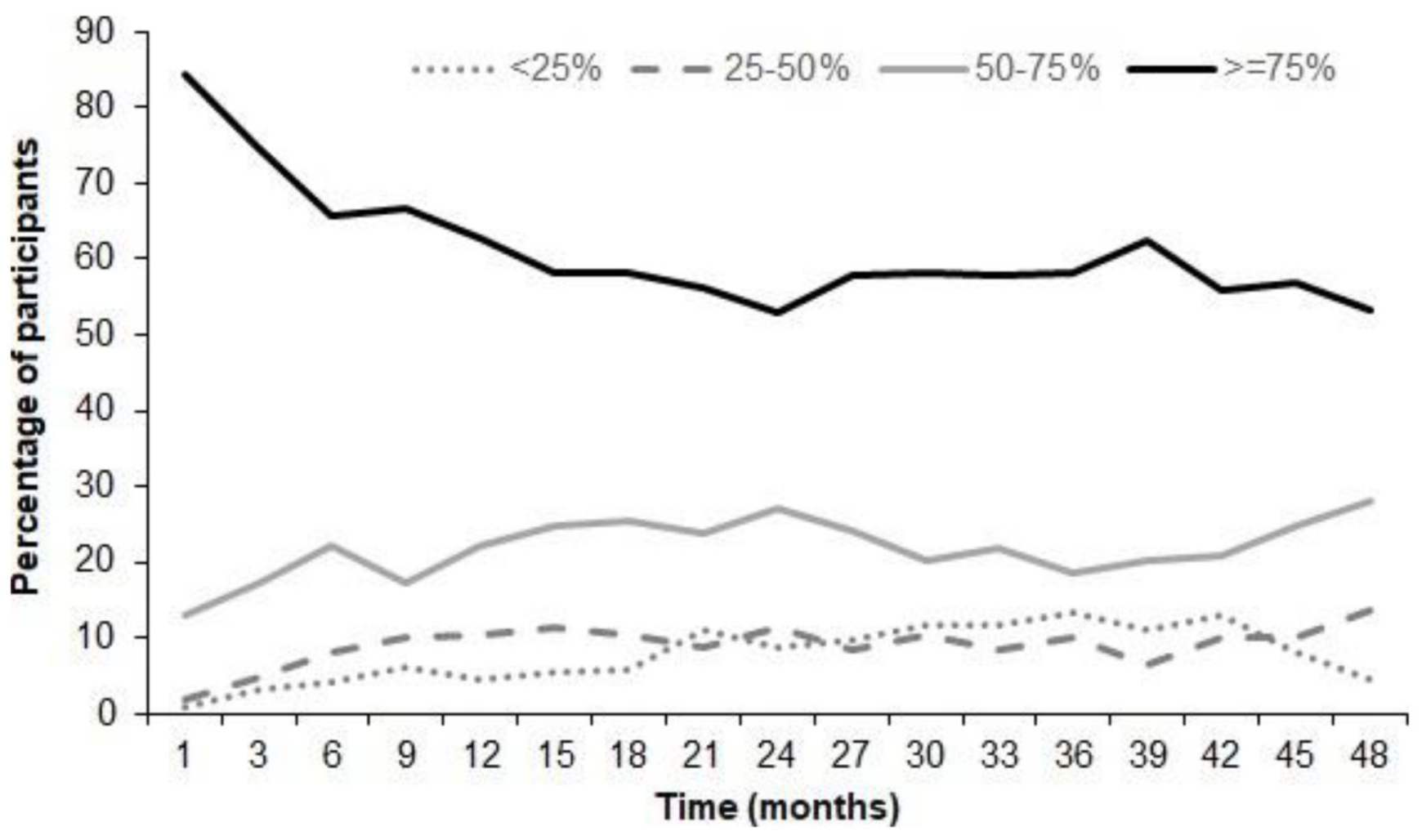

Figure 3.

Percentage of participants showing different levels of adherence, from $\geq 75 \%$ to $<25 \%$ 
Table 1

Baseline predictors of adherence during the trial

\begin{tabular}{|l|c|c|}
\hline & Beta coefficient & P-value \\
\hline Univariate linear regression models & & \\
\hline Sex (female vs male) & -0.04 & 0.41 \\
\hline Age (years) & -0.10 & 0.03 \\
\hline Age at diagnosis (years) & -0.007 & 0.88 \\
\hline Diabetes duration (years) & -0.05 & 0.34 \\
\hline HbA1c (mmol/mol) & -0.21 & $<0.001$ \\
\hline Diabetes treatment (MDI vs CSII) & 0.05 & 0.35 \\
\hline Country (Australia vs UK vs Canada) & -0.18 & $<0.001$ \\
\hline Multivariate linear regression model & & \\
\hline Age (years) & -0.09 & 0.05 \\
\hline HbA1C (mmol/mol) & -0.20 & $<0.001$ \\
\hline Country (Australia vs UK vs Canada) & -0.19 & $<0.001$ \\
\hline
\end{tabular}

Dependent variable is median adherence over time; independent variables are as collected at the baseline study visit. MDI: multiple daily injections; CSII: continuous subcutaneous insulin infusion 